\title{
Feasibility and Outcome of a Phase II Study of Intensive Induction Chemotherapy in 91 Elderly Patients with AML Evaluated Using a Simplified Multidimensional Geriatric Assessment
}

\author{
Debora Capelli - Francesco Saraceni - Alessandro Fiorentini • \\ Martina Chiarucci · Diego Menotti · Antonella Poloni · \\ Giancarlo Discepoli · Pietro Leoni · Attilio Olivieri
}

Received: February 3, 2020 / Published online: April 15, 2020

(C) The Author(s) 2020

\begin{abstract}
Introduction: We prospectively tested in a phase II study high-dose aracytin and idarubicin plus amifostine as induction regimen in 149 patients with acute myeloid leukaemia (AML) aged $\geq 60$ years, evaluated by a simplified multidimensional geriatric assessment (MGA).

Methods: Ninety-one fully or partially fit patients (61\%) were allocated to intensive chemotherapy and 58 (39\%) frail patients to best supportive care (BSC). Intensively treated patients, showing early death and complete response (CR) rate respectively of $5.5 \%$ and
\end{abstract}

Digital Features To view digital features for this article go to https://doi.org/10.6084/m9.figshare.12000042.

Electronic Supplementary Material The online version of this article (https://doi.org/10.1007/s12325020-01310-4) contains supplementary material, which is available to authorized users.

D. Capelli $(\varangle) \cdot$ F. Saraceni · A. Fiorentini . M. Chiarucci - D. Menotti - A. Poloni - P. Leoni . A. Olivieri

Hematology Department, University of Ancona, Azienda Ospedaliero Universitaria Ospedali Riuniti di Ancona, Via Conca 71, 60126 Ancona, Italy e-mail: debora.capelli@ospedaliriuniti.marche.it

\section{G. Discepoli}

Cytogenetic Laboratory, Azienda Ospedaliero

Universitaria Ospedali Riuniti di Ancona, Ospedale

Salesi, via F. Corridoni, 11, 60100 Ancona, Italy
$73.6 \%$, received 61 consolidations, followed by autologous transplant (ASCT), stem cell transplantation (SCT) or gemtuzumab ozogamicin, depending on mobilization outcome and donor availability.

Results: The 8-year overall survival (OS) of these patients was $20.4 \%$, with median duration of 11.4 months significantly superior to the 1.5 months of BSC arm $(p<0.001)$. Hyperleukocytosis and cytogenetics were predictors of survival with a relative risk of 1.8 in patients with poor karyotype without hyperleukocytosis $(p=0.02)$ and 3 in those with hyperleukocytosis $(\geq 50,000 / \mu \mathrm{l})(p=0.002)$.

Conclusion: MGA allowed tailored post-consolidation in $53.8 \%$ of patients after high-dose aracytin induction, with long-term survival doubling that reported in the literature after standard-dose cytarabine regimens.

Trial Registration: The study was registered with the Umin Clinical Trial Registry (www. umin.ac.jp/ctr), number R000014052.

Keywords: Acute myeloid leukaemia (AML); Autologous transplant (ASCT); Elderly; Gemtuzumab ozogamicin (GO); Multidimensional geriatric assessment (MGA); Oncology 


\section{Key Summary Points}

Why carry out this study?

This prospective phase II study evaluates the feasibility and efficacy of an intensive induction schedule, including high-dose aracytin plus idarubicin, preceded by amifostine, in a cohort of elderly patients with acute myeloid leukaemia, whose fitness was evaluated by simplified Balducci's multidimensional geriatric assessment. This subset of patients still represents an unmet medical need with $30-50 \%$ complete response rates and 5\% 10 -year overall survival probability.

This study aimed to demonstrate a survival advantage of $15 \%$ compared to the historical long-term data of $5 \%$. Secondary end points were disease-free survival, event-free survival, induction treatment-related death, haematological and non-haematological toxicities.

\section{What was learned from the study?}

This approach with intensified dose aracytin plus idarubicin in elderly patients with acute myeloid leukaemia, selected using a simplified multidimensional geriatric assessment, is feasible in $>60 \%$ of patients.

The survival rate was more than double and complete response rate was $20 \%$ higher than those reported in the literature, with low induction death rate (5\% vs $10-20 \%$ reported).

Multidimensional geriatric assessment identified frail patients ineligible for intensive therapy while fit and partially fit patients with acute myeloid leukaemia had similar tolerance and outcome apart from delay of polymorphonuclear neutrophil recovery $>1500 / \mathrm{ml}$ after induction (15 days in fit patients vs 21 days in partially fit patients, $p=0.03$ ).
Multidimensional geriatric assessment represents an accurate tool to define eligibility for chemotherapy since French Decisional Index and Sorror haematopoietic cell transplantation comorbidity index did not influence outcome and tolerance. Considering the very poor outcome of elderly patients, even in the new drugs era, this regimen represents an excellent backbone for future protocols, exploring new post-remission treatments in this unfavourable setting.

\section{INTRODUCTION}

The poor outcome of elderly patients with acute myeloid leukaemia (AML) is mainly due to the unfavourable (peculiar) biological properties of the disease and their frailty caused by comorbidities $[1,2]$ and the physiological process of ageing $[3,4]$. Consequently, only a minority of (elderly) patients aged over 60 receive adequate intensive induction treatment and very few can be enrolled in prospective clinical trials; moreover, the adverse biological characteristics of the disease $[5,6]$ including the high incidence of primary multidrug resistance phenotype are responsible for the lower complete response (CR) rate observed in this subset than younger patients [7]. To improve both the efficacy and the tolerance of chemotherapy, different approaches have been tested in this setting: the benefits of anthracycline dose escalation seem restricted to patients younger than 65 years [8]; fludarabine-containing regimens demonstrated better extra-haematological tolerance and an encouraging response rate, but without longterm control of the disease in a non-transplant setting [9]. Intensification of the cytarabine dose has been tested in younger patients with encouraging results [10]. Recent guidelines from the National Comprehensive Cancer Network (NCCN) and Italian Society of Haematology (SIE) [11, 12], however, discourage this approach in an elderly population because of the high extra-haematological toxicity. Preliminary reports show the feasibility and safety of 
high-dose cytarabine (HD-ARAC) and idarubicin in elderly patients with AML [13]. The clinical comparison between different kinds of anthracycline compounds yielded overlapping results [14], although a higher CR rate was recently observed with idarubicin in patients older than 50 [15]. We previously reported that a single high dose of idarubicin along with HDARAC and amifostine was feasible [13] in a preliminary series of 41 fit elderly patients with AML. Moreover Charlson Comorbidity Index, Autonomy Daily Life (ADL) scores and geriatric syndromes were shown to predict response, mortality and survival in elderly patients with AML [16-18]. On the basis of this background we planned to assess the real feasibility and effectiveness of a treatment tailored on a simplified Balducci's multidimensional geriatric assessment (MGA) [19], in a large cohort of 149 elderly patients with AML, evaluated according to a method previously tested in patients with diffuse large B cell lymphoma (DLBCL) [20].

\section{METHODS}

This phase II single-centre non-randomized study aimed to prospectively evaluate the feasibility and outcome of intensive induction chemotherapy in an unselected series of elderly patients, observed in real-life practice.

Between June 1999 and September 2010 we prospectively observed 149 consecutive patients with non-M3 AML over 59 years (range 60-89), who were evaluated using a simplified MGA, as reported elsewhere $[17,19]$ and in the supplementary material. Difficulties in performing tests, due to AML symptoms and cultural differences, might impair the results of tests for cognitive and instrumental abilities and we decided to evaluate only comorbidities, geriatric syndromes and physical functions. This allowed us to identify three groups of patients: (1) fit patients $(N=70,47 \%)$ comprising those with ADL score $=6$, no grade II comorbidities and geriatric syndromes; (2) partially fit patients $(N=21,14 \%)$ with $\mathrm{ADL}=6,<3$ grade II comorbidities, no geriatric syndromes who fulfilled all the other inclusion criteria (Eastern Cooperative Oncology Group (ECOG)/World
Health Organisation (WHO) performance status $\leq 2$, serum bilirubin $<1.5$ ULN and creatinine below normal; ejection fraction $\geq 45 \%$, absence of other malignancies) and received intensive chemotherapy; (3) frail patients $(N=58,39 \%)$, with ADL $<6$ or 1 grade III or $>2$ grade II comorbidities or a geriatric syndrome not related to AML or aged $>85$ years, were considered "a priori" eligible only for best supportive care (hydroxyurea or vinblastine and transfusion support). MGA was part of eligibility criteria: 42/58 frail patients met the inclusion criteria but were considered frail and so not enrolled. The main causes of frailty are reported in the supplementary material.

Secondary patients with AML and history of environmental, occupational or therapeutic exposure to haematotoxins or radiation or evolving from at least 6 months antecedent myelodysplasia or other myeloid stem cell disorders were also enrolled.

Cytogenetic risk was available in 81 patients, determined by applying the Southwest Oncology Group (SWOG)/ECOG classification [21].

\section{Compliance with Ethics Guidelines}

This research involved human participants. All patients gave written informed consent, according to the Declaration of Helsinki. Additionally, the Ethics Committee of Ospedali Riuniti di Ancona approved the protocol. The study was registered with the Umin Clinical Trial Registry (www.umin.ac.jp/ctr), number R000014052.

\section{Induction Chemotherapy}

Ninety-one eligible patients (61\%), of the 149 screened by MGA, received intensive induction chemotherapy, the so-called Memorial, consisting of $3000 \mathrm{mg} / \mathrm{m}^{2}$ per day of cytarabine by 3 -h infusion for 5 days and $40 \mathrm{mg} / \mathrm{m}^{2}$ of idarubicin given intravenously on day 3 for $20 \mathrm{~min}$, preceded by $1000 \mathrm{mg}$ of intravenously administered amifostine as described elsewhere [13].

In patients older than 70 years the idarubicin and cytarabine doses were reduced by $25 \%$. Supportive care, timing of bone marrow 
biopsies and response criteria were the same as previously reported [13].

\section{Consolidation Chemotherapy and Post- Consolidation Strategy}

Sixty-one patients received an HD-ARAC consolidation scheme reduced to 4 days with a further $25 \%$ reduction of the dose in patients older than 70 years $(N=15)$. PBSC (peripheral blood stem cell) collections were planned after consolidation therapy and filgrastim at $5 \mu \mathrm{g} / \mathrm{kg}$ per day was administered subcutaneously from day +4 after the end of chemotherapy until the last leukapheresis. A minimum dose of $\mathrm{CD} 34^{+}$ cells collected $\left(>3 \times 10^{6} / \mathrm{kg}\right)$ was required for a post-consolidation autologous transplant (ASCT); where the PBSC harvested was insufficient a second mobilization attempt was allowed, after a second course of chemotherapy, including a VP-16 plus cytosine-arabinoside (ARA-C) intermediate dose for 3 days, followed by granulocyte colony-stimulating factor (GCSF). Poor mobilizers and patients ineligible for ASCT for other reasons received gemtuzumab ozogamicin (GO) consolidation of 3 monthly $3 \mathrm{mg} / \mathrm{m}^{2}$ i.v. infusions followed by three further courses every 3 months as previously described [22]. Relapsed patients received a second-line chemotherapy at the clinician's discretion.

\section{Response and Outcome Definitions}

Response criteria and treatment outcomes were defined according to the recommendations of Cheson et al. [23]. Also see the supplementary material.

\section{Statistical Methods}

The primary end point was initially the improvement of the CR rate; the study was then amended including overall survival (OS) as primary end point, disease-free survival (DFS), event-free survival (EFS), induction treatmentrelated death (TRD), haematological and nonhaematological toxicities as secondary end points. The initial sample size was 42 patients, calculated according to the Simon minimax design [24], predicting a 20\% increase in the CR rate, in comparison with the current gold standard of 50\% reported in the literature (beta and alpha errors of 0.20 and 0.05 , respectively) $[8,25]$. We then extended the sample size to 91 patients, in order to demonstrate a survival advantage of $15 \%$ compared to the historical long-term data of $5 \%[8,18,26,27]$, with the same alpha and beta errors. The planned time of accrual was 11 years with 5 years follow-up.

In addition to the main prognostic factors, we evaluated the French Decisional Index (FDI) [28] and the haematopoietic cell transplantation co-morbidity index (HCT-CI) [29] as variables potentially influencing outcome (Table $1 \mathrm{~s}$ in the supplementary material).

The two-sided Fisher's exact test was performed to analyse the influence of patient and disease characteristics (age $>70$ years, cytogenetic risk, WBC count $>50,000 / \mu \mathrm{l}$, ECOG/ WHO performance status (PS), secondary AML, FDI and HCT-CI) on the response rate. The distribution of OS, DFS and EFS was estimated by the Kaplan-Meier method [30]. Log rank test was performed to compare survival probabilities by age group and disease characteristics. All factors identified at univariate analysis with a $p$ value less than 0.1 were further analysed by Cox regression multivariate analysis. All test results are reported using two-sided $p$ values for which 95\% confidence interval (CI) hazard ratios were calculated.

SPSS 18.0 software was used to analyse the data.

\section{RESULTS}

\section{Baseline Characteristics}

The baseline characteristics of the three groups of patients are listed in Table 1. Frail patients were older, had a poorer PS score and significantly more secondary and poor prognostic karyotype AML than the fit and partially fit subgroup of patients. Partially fit patients had worse HCT-CI score $(47.6 \%$ with score $>2)$ compared to fit patients $(12.8 \%$ with score $>2)$ $(p=0.0001)$. All the other baseline 
Table 1 Patients characteristics of the 91 intensively treated (70 fit and 21 partially fit) and 58 BSC (best supportive care) patients

\begin{tabular}{|c|c|c|c|c|c|c|c|}
\hline & $\begin{array}{l}\text { Intensive induction } \\
\text { Fit/partially fit, } \\
N(\%)\end{array}$ & $\begin{array}{l}\text { BSC } \\
N(\%)\end{array}$ & $p$ & & $\begin{array}{l}\text { Intensive induction } \\
\text { Fit/partially fit, } \\
N(\%)\end{array}$ & $\begin{array}{l}\text { BSC } \\
N(\%)\end{array}$ & $p$ \\
\hline Gender & & & 0.87 & $\begin{array}{l}\text { Performance } \\
\text { status }\end{array}$ & & & 0.003 \\
\hline Male & $39 / 11(55.7 / 55)$ & $31(53.4)$ & & $0-1$ & $64 / 16(91.4 / 76.2)$ & $32(55.6)$ & \\
\hline Female & $31 / 10(44.3 / 47.6)$ & $27(46.6)$ & & 2 & $4 / 3(5.7 / 14.3)$ & $26(44.4)$ & \\
\hline Karyotype & & & 0.05 & 3 & $2 / 2(2.8 / 9.5)$ & 0 & \\
\hline Favourable & $5 / 0(7.1 /-)$ & 0 & & FDI & & & 0.49 \\
\hline Intermediate & $31 / 12(44.3 / 57.1)$ & $11(35.5)$ & & 0 & $40 / 12(57.1 / 57.1)$ & $4(66.7)$ & \\
\hline Unfavourable & $25 / 8(35.7 / 38.1)$ & $20(64.5)$ & & $>0$ & $30 / 9(42.9 / 42.9)$ & $2(33.3)$ & \\
\hline AML type & & & 0.005 & HCT-CI & & & \\
\hline $\begin{array}{l}\text { De novo } \\
\text { AML }\end{array}$ & $42 / 13(60 / 62)$ & $27(46.5)$ & & $0-2$ & $48 / 11(68.6 / 55)$ & $5(55.6)$ & 0.23 \\
\hline $\begin{array}{l}\text { Secondary } \\
\text { AML }\end{array}$ & $28 / 8(40 / 38)$ & $31(53.4)$ & & $>2$ & $9 / 10(12.8 / 47.6)^{*}$ & $2(44.4)$ & \\
\hline Age & & & $<0.001$ & WBC count & & & 0.45 \\
\hline$<70$ years & $41 / 11(58.6-52.4)$ & $10(17.2)$ & & $<50,000 / \mu \mathrm{l}$ & $61 / 19(87.2 / 90.5)$ & $48(82.4)$ & \\
\hline$>69$ years & $29 / 10(41.4 / 47.6)$ & $48(82.8)$ & & $\geq 50,000 / \mu \mathrm{l}$ & $9 / 2(12.8 / 9.5)$ & $10(17.6)$ & \\
\hline $\begin{array}{r}\text { Median } \\
\text { (range) }\end{array}$ & $68(60-77)$ & $\begin{array}{l}78 \\
\quad(60-89)\end{array}$ & & & & & \\
\hline
\end{tabular}

FDI French Decisional Index, HCT-CI haematopoietic cell transplant-comorbidity index ${ }^{*} p<0.0001$

characteristics were equally distributed between fit and partially fit patients.

\section{Toxicity}

Haematological and extra-haematological toxicities were assessed in 91 and 61 patients after induction and consolidation respectively and are reported in Table 2 separately in fit and partially fit patients. After induction we observed five $(5.5 \%)$ TRDs: one patient died of pneumonitis, two of febrile neutropenia and two of microbiologically documented infections; we observed six TRDs during consolidation therapy (9.2\%): five from infectious episodes and one death while in aplasia.
We did not observe excess toxicity in partially fit patients apart from longer polymorphonuclear neutrophil (PMN) recovery $>1500$ / $\mu \mathrm{l}$ after induction (15 days in fit patients vs 21 days in partially fit patients, $p=0.03$ ) which did not translate into longer duration of hospitalization or higher incidence of grade III-IV infection or neutropenic fever.

The median time required to achieve a neutrophil count $>1500 / \mu \mathrm{l}$ was similar after consolidation: 14 days in fit vs 17.5 days respectively in the two groups.

Platelet recovery, transfusional need, extrahaematological toxicity, median duration of fever $>38^{\circ} \mathrm{C}$, i.v. antibiotic therapy, 
Table 2 Haematological, extra-haematological toxicity and transfusional need after 91 induction and 61 consolidation courses in fit and partially fit patients

\begin{tabular}{|c|c|c|c|c|}
\hline & \multicolumn{2}{|l|}{ Induction } & \multicolumn{2}{|c|}{ Consolidation } \\
\hline & Fit $(n=70)$ & $\begin{array}{l}\text { Partially fit } \\
(n=21)\end{array}$ & Fit $(n=47)$ & $\begin{array}{l}\text { Partially fit } \\
(n=14)\end{array}$ \\
\hline Toxicity-related death, $N(\%)$ & $4(5.7)$ & $1(4.7)$ & $6(12.7)$ & 0 \\
\hline $\begin{array}{l}\text { Neutrophil }>1500 / \mu l \text {, median day of recovery } \\
\text { (range) }\end{array}$ & $15(9-33)$ & $21(12-72)^{*}$ & $14(9-53)$ & $17.5(13-23)$ \\
\hline $\begin{array}{l}\text { Platelet }>20,000 / \mu 1, \text { median day of recovery } \\
\quad(\text { range })\end{array}$ & $16.5(12-26)$ & $19(12-36)$ & $15(8-49)$ & $16.5(12-32)$ \\
\hline $\begin{array}{l}\text { Platelet }>100,000 / \mu \mathrm{l}, \text { median day of recovery } \\
\quad \text { (range) }\end{array}$ & $17(11-52)$ & $21.5(13-43)$ & $29(12-68)$ & $25.5(15-50)$ \\
\hline Red blood cell transfusions, median (range) & $11(3-32)$ & $10(4-47)$ & $6(0-24)$ & $4(2-10)$ \\
\hline Platelet transfusions, median (range) & $6(0-22)$ & $6(2-19)$ & $5(0-24)$ & $3(1-12)$ \\
\hline Grade III-IV mucositis incidence, $N(\%)$ & $2(2.9)$ & $2(9.5)$ & $7(15)$ & $1(4.7)$ \\
\hline Grade III-IV hepatic toxicity incidence, $N(\%)$ & $7(10)$ & $1(4.7)$ & $8(17)$ & $2(14.3)$ \\
\hline Grade III-IV cardiac toxicity incidence, $N(\%)$ & $1(1.4)$ & - & $2(4.2)$ & $1(7)$ \\
\hline $\begin{array}{l}\text { Grade III-IV neurologic toxicity incidence, } \\
\quad N(\%)\end{array}$ & $1(1.4)$ & - & $3(6.4)$ & - \\
\hline $\begin{array}{l}\text { Grade III-V infections/febrile neutropenia, } \\
\quad N(\%)\end{array}$ & $\begin{array}{r}33(47) / \\
14(20)\end{array}$ & $10(47) / 9(42)$ & $\begin{array}{c}30(63.8) / \\
4(8.5)\end{array}$ & $6(28.6) / 1(7)$ \\
\hline Duration of fever, median (range) & $5.5(0-27)$ & $4(0-11)$ & $2(0-15)$ & $2.5(0-11)$ \\
\hline Duration of antibiotic, median (range) & $16(0-66)$ & $18(0-34)$ & $10(0-32)$ & $9(0-21)$ \\
\hline Duration of hospitalization, median (range) & $30(15-56)$ & $32(24-61)$ & $24(14-61)$ & $24(16-40)$ \\
\hline
\end{tabular}

${ }^{*} p=0.03$

hospitalization after induction and consolidation therapy were similar between the two groups.

Details of febrile episodes are reported in the supplementary material.

\section{Response}

We observed 67 CRs (76.1\%) in the 88 patients whose response was assessable (3 patients who died during aplasia were too early for evaluation); 21 had a resistant disease. Consistent with the intention to treat (ITT) criterion, the CR rate was $73.6 \%$.
Fit patients along MGA criteria had $71.4 \%$ CR rate similar to that of partially fit patients (81\%) (Table $2 \mathrm{~s}$ in the supplementary material).

Patients with unfavourable cytogenetics achieved a $63.6 \%$ CR rate, significantly lower than the $85.1 \%$ observed in patients with favourable- and intermediate-risk cytogenetic AML $(p=0.03)$; in the 35 patients with antecedent secondary AML, the CR rate was $65.7 \%$. The CR rate in the 39 patients with FDI $>0$ was $66.7 \%$. Cytogenetic risk, gender, secondary disease diagnosis and FDI entered the multivariate analysis: poor-risk cytogenetics was the only factor able to significantly predict a lower CR rate (RR of 3.26; 95\% CI 1.18-9.53). 
Among the 67 patients in CR, 61 (91\%) received at least one consolidation course.

Early relapse $(n=3)$, death in CR $(n=1)$ or ineligibility due to poor PS $(n=2)$ ruled out the possibility of first consolidation therapy. After consolidation we observed six toxicity-related deaths and two early relapses, while there was a severe deterioration in the PS of four patients who were consequently forced to drop out of the protocol. Forty-nine patients, therefore, underwent PBSC mobilization; 24 of them (49\%) achieved successful mobilization. Lastly, 22 patients received ASCT; among the 24 patients failing PBSC mobilization, 23 received post-consolidation therapy based on GO and 4 underwent allogeneic transplant because of high risk of the disease.

\section{OS, DFS and EFS}

With a median follow-up of 70 months (range 24-124) 20 patients are still alive; 17 are still in conventional care regimens (CCR) while 3 relapsed, but achieved a second CR after salvage treatment. One is still in second CR, and the other two eventually relapsed after retreatment with GO. The 8-year OS, DFS and EFS were respectively $20.4 \% \quad(11.1-34.8 \%), \quad 22.9 \%$
(14.5-34.2\%) and 17.9\% (11.3-27.2) (Fig. 1a). Median OS, DFS, EFS were 11.4 and 7.8 and 7 months respectively. The OS of patients identified as frail was significantly lower since all died within 18 months with a median OS of 1.5 months $(p<0.001$, Fig. $1 b)$, while similar survival was observed in fit and partially fit patients (Table $3 \mathrm{~s}$ in the supplementary material).

The 8-year OS was significantly reduced in patients with a secondary disease vs those with de novo AML (10.4\% vs $27.1 \% ; p=0.04)$, reduced in those with unfavourable-risk cytogenetics vs other risk groups $(11.4 \%$ vs $30.3 \%$ $p=0.01)$ and reduced in patients with a WBC count $>50,000 / \mu \mathrm{l} \quad \mathrm{vs}<50,000 / \mu \mathrm{l} \quad(0 \% \quad$ at 33 months vs $23.4 \%, p=0.009)$. Similar results were observed for DFS and EFS. Furthermore, patients older than 69 years showed both a lower DFS (10\% vs $33.3 \%, p=0.04)$ and EFS $(7.7 \%$ vs $26.2 \%, p=0.05)$ than younger patients.

Multivariate analysis confirmed the prognostic value of cytogenetic risk and hyperleukocytosis (Table 3). Patients with poor karyotype had a 2.1 relative risk of dying or a 1.89 relative risk of dying or relapse when compared with patients with intermediate- and

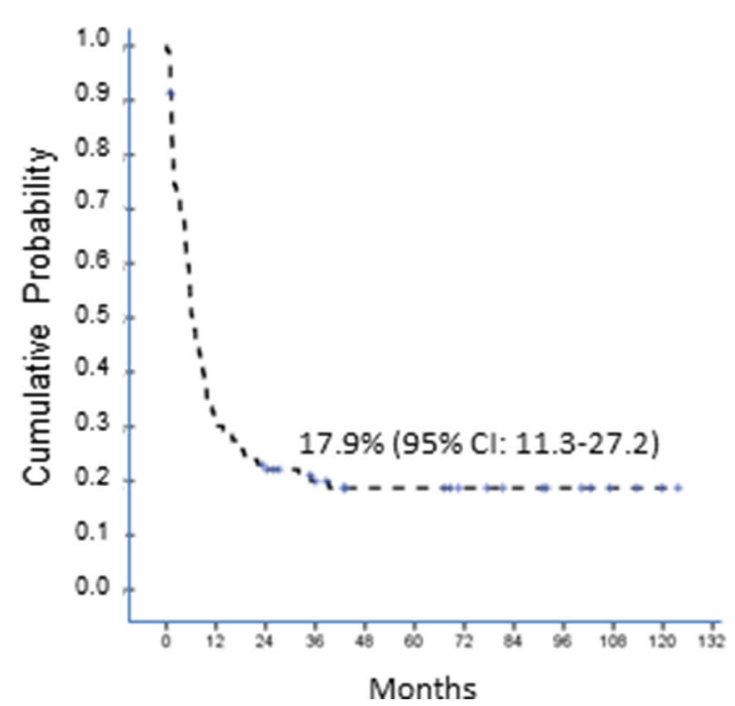

(a)

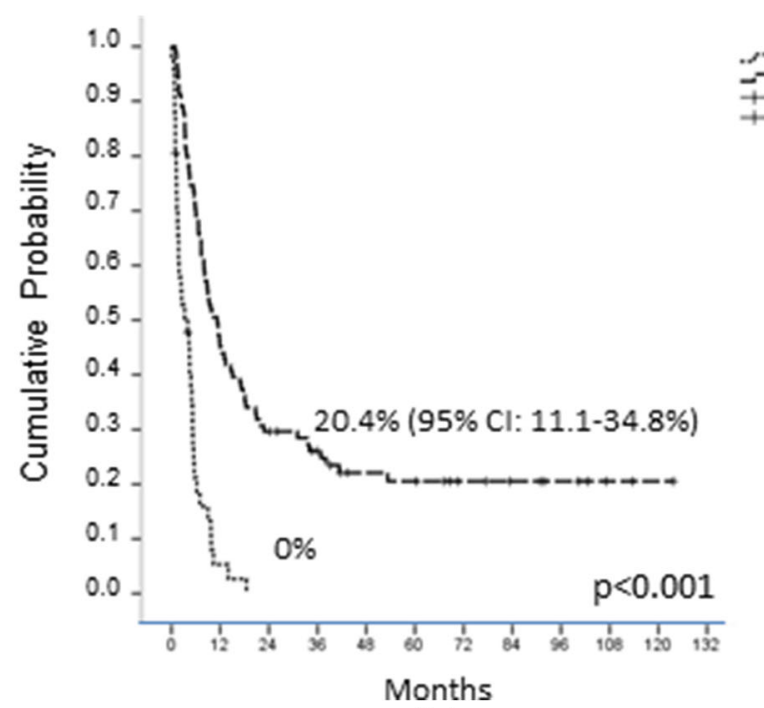

(b)

Fig. 1 a EFS in 91 fit and partially fit patients with AML receiving intensive treatment (Memorial induction). b OS in 149 patients with AML, 91 fit and partially fit receiving Memorial induction and 58 frail receiving best supportive care (BSC) 


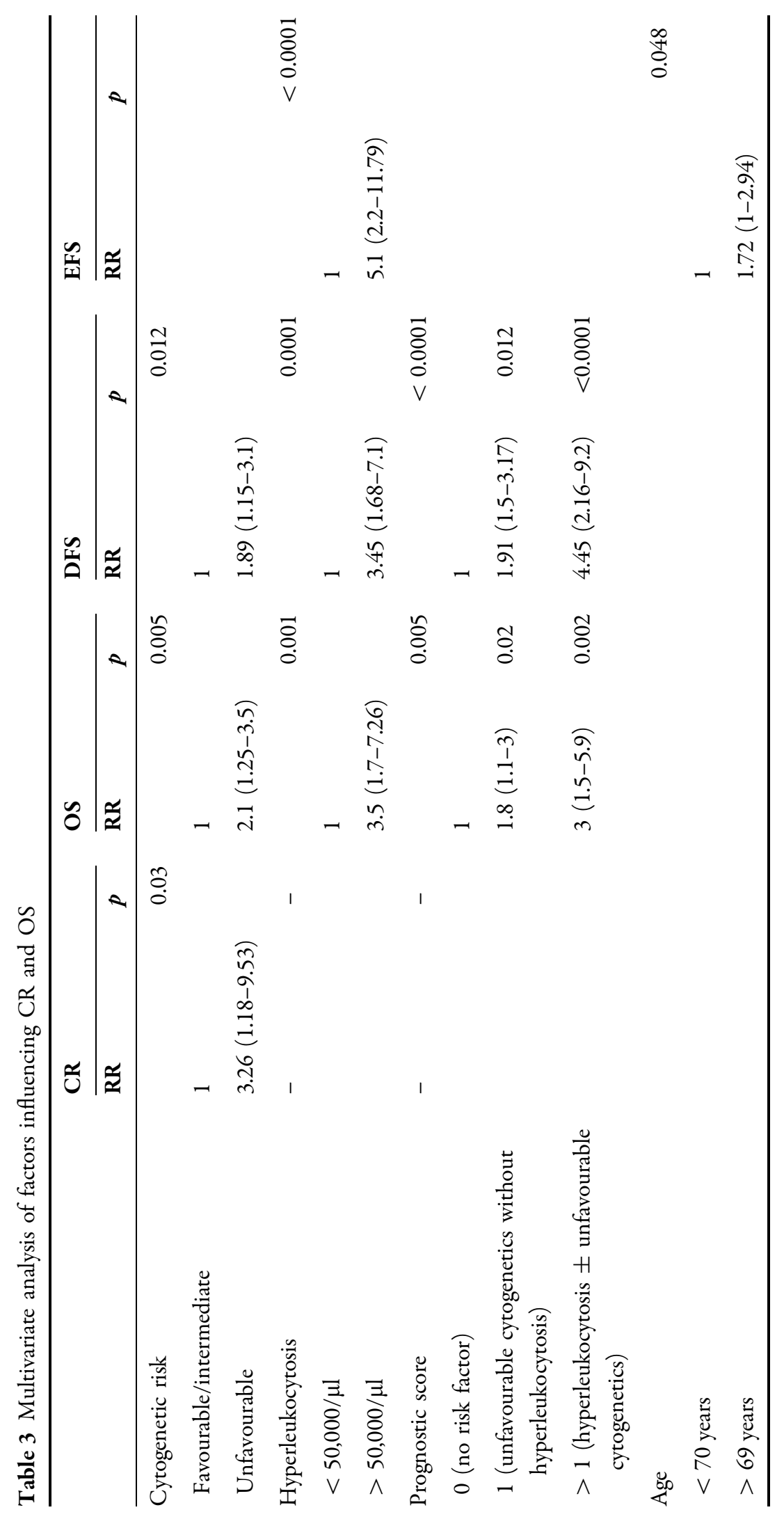




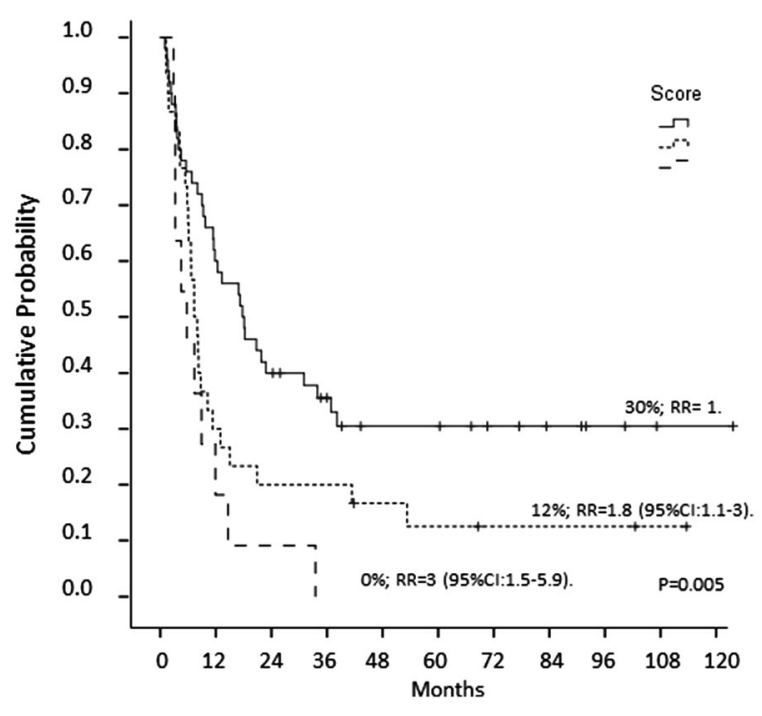

Fig. 2 OS in 91 elderly patients with AML by prognostic score. Score $=0$ : WBC $<50,000 / \mathrm{ml}$, favourable $/$ intermediate cytogenetics. Score $=1$ : WBC $<50,000 / \mathrm{ml}$, unfavourable cytogenetics. Score $>1$ : $\quad$ WBC $>50,000 /$ $\mathrm{ml} \pm$ unfavourable cytogenetics

low-risk cytogenetics. Hyperleukocytosis $(\geq 50,000 / \mu \mathrm{l})$ at the time of diagnosis significantly lowered OS and EFS, with relative risks respectively of 3.53 and 3.45 . We tested a prognostic score assuming that the presence of unfavourable karyotype and hyperleukocytosis accounts for a score of 1 and 2, respectively. We therefore identified three groups of patients: one with no risk factor (score $=0$ ) and 30\% 8 -year OS, RR of dying of 1 ; one with unfavourable karyotype alone (score =1) $1.8 \mathrm{RR}$ of dying (95\% CI 1.1-3, $p=0.02$ ) and 12\% 8-year OS; one with hyperleukcytosis alone or with unfavourable karyotype (score $>1$ ) with 3 RR of dying (95\% CI 1.5-5.9, $p=0.002)$ and 0\% 3-year OS (Table 3, Fig. 2).

\section{DISCUSSION}

The cure rate for AML in the elderly is very low and the outcome for these patients is generally poor. Unfortunately, very few prospective trials in this setting address the outcome of an unselected population of elderly patients with AML, as only a minority of them are enrolled in clinical trials $[3,31]$.
On the basis of our previous encouraging experience in the setting of aggressive lymphoma [16], we describe a simplified MGA as a tool for selection of elderly patients with AML eligible for intensive treatment, which identified $39 \%$ of the patients with AML referred to our centre as frail and suitable only for BSC while $61 \%$ satisfied the inclusion criteria for receiving intensive treatment.

As induction therapy we adopted the same schedule previously used, with encouraging results in the setting of relapsed/refractory acute lymphoblastic leukaemia, including HD-ARA-C plus single high-dose idarubicin [32], modified by incorporating amifostine, in order to reduce both haematological and extra-haematological toxicity [33-35]. Even though HD-ARA-C is not generally recommended as induction treatment, in the young and elderly alike [36], some studies [37] suggest that escalation of HD-ARA$C$ in combination with idarubicin could be effective both in induction and in consolidation treatment [10, 37, 38].

As suggested by our previous pilot experience [13], our schedule was feasible in this large series of patients with AML (both fit and partially fit, selected by the MGA) and was characterized by an excellent tolerability profile, with a 5\% induction death achieving an outstanding $73.6 \% \mathrm{CR}$ rate, according to the ITT criterion. Conversely, in the same subset of patients recent prospective studies also show an average CR rate of $50 \%$ with a $15 \%$ TRD and a long-term survival of $10 \%$ which is the half of that reported in our study [39]. Patients with poor cytogenetic AML have the worst prognosis in the over 60 s with a $26 \%$ CR rate in Grimwade et al.'s MRC analysis of 1065 elderly patients, and even if a direct comparison is not possible our CR rate doubled this result with $63.6 \%$ CR rate [6].

Induction schedules including updosing daunorubicin [8] or administering a low or fractionated dose of GO [40] failed to give similar results. The HOVON/SAKK/AMLSG randomized trial exploring the updosing of daunorubicin to $90 \mathrm{mg} / \mathrm{m}^{2}$ in combination with standard-dose ARA-C produced a $64 \%$ double induction CR rate with an 11\% 30-day mortality and a survival and response benefit only in the 
elderly up to 65 years. Furthermore, updosing daunorubicin failed to produce any survival benefit in patients with poor-risk cytogenetic AML.

To the best of our knowledge the best experience with high dose aracytin $\left(2 \mathrm{~g} / \mathrm{m}^{2}\right.$ per day for 6 days) combined with standard-dose daunorubicin produced a $69 \%$ CR rate and 10\% toxicity-related deaths [41] in the elderly setting with a median OS of 15.3 months, higher than that reported in our study (11.4 months). However our patients had higher transplant rate than that of Arellano et al.'s (28.9\% vs $10 \%)$, and consequently transplant mortality might have reduced first-year survival. Comparison between the long-term outcomes is unfortunately impossible because of the lack of longterm survival probability in Arellano et al.'s study.

Other studies exploring HD-ARAC feasibility and efficacy in similar settings included fludarabine \pm gemtuzumab and idarubicin with a similar toxicity profile but lower CR rate than our and Arellano et al.'s studies, probably because of a 33\% higher dose of cytarabine used in the latter compared to FLAG-like schemes [42-44] (Clavio, Candoni, Ferrara).

A retrospective analysis of experiences at $\mathrm{M}$. D. Anderson Cancer Center (MDACC) described outcomes of 446 patients with AML aged $\geq 70$ years receiving various intensive cytarabine-based regimens (up to $2 \mathrm{~g} / \mathrm{m}^{2}$ daily) including fludarabine, idarubicin or other drugs. They reported a CR of $45 \%$ with a median OS of 4.6 months and early 8-week mortality rates of $36 \%$ [45]. The presence of at least one of the factors age $>80$ years, PS $\geq 2$ (ECOG/ WHO), complex karyotype ( $\geq 3$ abnormalities) and creatinine level $>1.3 \mathrm{mg} / \mathrm{dl}$ predicted high 8 -week mortality rate, which was reduced when low air flow room facilities were available with a hazard ratio of 0.35 .

In our population the presence of a previous haematological disease and an FDI score $>1$ negatively affected the response, but the unfavourable-risk cytogenetics was the only negative predicting response factor. However, it should be underlined that in those patients with unfavourable karyotype, secondary AML or FDI $>1$ the CR rate was equally good: $63.6 \%$,
$65.7 \%$ and $66.7 \%$, respectively. In a similar setting, clofarabine achieved a lower CR rate, ranging from $40 \%$ to $30 \%$ in fit and partially fit elderly patients, respectively $[46,47]$.

Hypomethylating agents might represent an interesting and attractive alternative option, with better quality of life, in comparison to conventional chemotherapy. A multicentre randomized study evaluated azacitidine (AZA) efficacy and safety vs CCR in 488 patients with AML, aged $\geq 65$ years, with $>30 \%$ bone marrow blasts [48]. The study demonstrated a 1-year survival superiority of AZA over CCR with median OS of 12.1 months, comparable to ours, but a shorter median follow-up of 24 months. Similar efficacy was also observed in the extremely poor prognosis setting of unfavourable cytogenetic AML with median OS of 6.4 months in the AZA arm, compared to 7.3 months observed in our study. Unfortunately, the difference between the median RFS and OS (9.3 vs 10.6 months) was 1 month in the azacitidine arm and we did not find studies demonstrating clinical efficacy of new drugs in patients failing to respond to hypomethylating agents, who still represent an unmet medical need. A recent phase III study showed similar but inferior results after decitabine, another hypomethylating agent, with median OS of 7.7 months (3.1 months in poor cytogenetic risk setting) [49].

We also prospectively tested the feasibility in the real-life setting of an intensive program in a population of elderly patients with AML selected by MGA: overall $54 \%$ of patients (49/91) completed the program: according to the ITT criteria the 8-year overall survival was $20.4 \%$, with a median OS of 11.4 months These data compare well to the results reported by the EORTC and CETLAM groups where the postconsolidation strategy was represented by ASCT $[50,51]$. In less than half of the patients receiving consolidation chemotherapy who failed PBSC mobilization, not eligible for ASCT, we offered an alternative post-consolidation in the form of GO therapy with a very encouraging outcome [20]. One shortcoming of the study is its lack of molecular data on NPM and FLT3 status, even though their prognostic role in 
elderly patients setting is still controversial $[52,53]$.

Poor-risk cytogenetics and hyperleukocytosis were the only factors predicting OS. All patients with a WBC count $>50,000 / \mu \mathrm{l}$ died within 3 years and only $11.4 \%$ of patients with AML with poor cytogenetics were still alive at 8 years. FDI did not have any prognostic influence on survival and $15 \%(6 / 39)$ of patients with a score $>0$, defined by Malfuson as not eligible for intensive treatment with intention to cure [28], were actually cured by this HD-ARA-Cbased protocol. The presence of previous haematological malignancies was not a prognostic factor for poor OS in multivariate analysis, even though only $10.4 \%$ of patients were alive at 8 years.

The majority of scores predicting response and survival in elderly patients with AML include cytogenetics and leukocytosis, but the assessment of an accurate comorbidity score might help the evaluation of the risk-benefit balance of intensive treatment. CCI [14] and HCT-CI [54] are respectively associated with remission rate and survival in two recent retrospective studies, the latter performed in a younger population of median age 40 years. We prospectively selected elderly patients with AML by simplified MGA with comprehensive evaluation of physical functions and comorbidities.

HCT-CI was $>2$ in $47.6 \%$ of partially fit vs $12.8 \%$ of fit patients. These patients showed $65 \%$ CR rate and $21.1 \%$ 8-year OS, statistically similar to the $82.2 \% \mathrm{CR}$ rate and the $22 \% 8$-year OS observed in patients with HCT-CI $<3$. These results could be explained by the fact that the diagnosis of previous cancer accounts for 3 in HCT-CI, overestimating the score in some of the patients with previous history of cured neoplasia. Furthermore spirometry and DLCO (carbon monoxide diffusing capacity of the lung) normal values reported by Sorror et al. are rarely found in the elderly population, also producing an increasing of HCT-CI in this setting. The Sorror score might therefore underestimate eligibility for intensive treatment of elderly patients with AML. Balducci MGA instead computes active comorbidities, ADL and geriatric syndromes, thereby producing a reliable measure of patient fitness. We previously published data on MGA tailored treatment in elderly patients with non-Hodgkin lymphoma, who received reduced dosages if partially fit. The initial AML study, performed in 42 patients, showed feasibility of intensive treatment even in partially fit elderly patients, probably owing to the use of the cytoprotector agent amifostine. We have therefore reduced the dosages only in patients over 70 as previously described. Partially fit patients showed $81 \% \mathrm{CR}$ rate and $28.6 \%$ 8-year OS similar to $71.4 \%$ CR rate and $18.2 \%$ 8-year OS observed in fit patients. These results confirm treatment feasibility in partially fit patients. As expected, the 58 patients with AML identified as frail by the MGA, receiving BSC, achieved a median OS of 1.5 months.

\section{Study Limitations}

This is not a retrospective case-control study and we can only compare our results to those reported in the literature in similar settings with standard-dose ARA-C, GO, fludarabine, intensified daunorubicine-containing regimens. We reduced dosages of chemotherapy drugs only in patients over 70 and not in younger partially fit patients.

\section{CONCLUSION}

This approach with aggressive induction in elderly patients with AML selected using the simplified MGA is feasible in $>60 \%$ of elderly patients with AML and was highly effective in terms of the CR rate, without relevant treatment-related mortality. Despite the delay of PMN recovery $>1500 / \mu \mathrm{l}$ after induction in the partially fit patients with AML ( 15 days in fit patients vs 21 days in partially fit patients, $p=0.03$ ) we did not observe longer duration of hospitalization, higher incidence of grade III-IV infection, neutropenic fever and induction death rate, suggesting the optimal tolerance of this schedule in comparison to fit patients. Moreover, $>50 \%$ of the patients in CR were able to receive an effective consolidation regimen, with an encouraging outcome: indeed, the survival rate was more than double that 
reported in the literature in a similar setting $[17,38,55,56]$. This regimen might therefore provide an excellent backbone for future protocols, exploring new post-remission treatments in this unfavourable setting.

MGA assessment might guide the enrollment in intensive treatment protocols, reducing the bias of patients selection in and between clinical trials. Simplified Balducci MGA was shown to be a reliable measure of functional status and comorbidities when compared to HCT-CI score in this monocentric study. Its reliability and reproducibility should be validated in larger multicentre studies.

\section{ACKNOWLEDGEMENTS}

We would like to thank patients who participated in our study. The authors also wish to thank Paul Bowerbank for his help in reviewing the English of the manuscript.

Funding. This study was supported by AIL of Ancona. All authors had full access to all of the data in this study and take complete responsibility for the integrity of the data and accuracy of the data analysis. No Rapid Service Fee or Open Access Fee was received by the journal for the publication of this article.

Authorship. The authors meet the International Committee of Medical Journals Editors (ICMJE) criteria for authorship for this article, they take responsibility for the integrity of the work as a whole, and have given their approval for this version to be published.

Disclosures. Debora Capelli, Francesco Saraceni, Alessandro Fiorentini, Martina Chiarucci, Diego Menotti, Antonella Poloni, Giancarlo Discepoli, Pietro Leoni, Attilio Olivieri have nothing to disclose.

Compliance with Ethics Guidelines. This research involved human participants. All patients gave written informed consent, according to the Declaration of Helsinki. Additionally, the Ethics Committee of Ospedali
Riuniti di Ancona approved the protocol. The study was registered with the Umin Clinical Trial Registry (www.umin.ac.jp/ctr), number R000014052.

Data Availability. The datasets generated during and/or analyzed during the current study are available from the corresponding author on reasonable request.

Open Access. This article is licensed under a Creative Commons Attribution-NonCommercial 4.0 International License, which permits any non-commercial use, sharing, adaptation, distribution and reproduction in any medium or format, as long as you give appropriate credit to the original author(s) and the source, provide a link to the Creative Commons licence, and indicate if changes were made. The images or other third party material in this article are included in the article's Creative Commons licence, unless indicated otherwise in a credit line to the material. If material is not included in the article's Creative Commons licence and your intended use is not permitted by statutory regulation or exceeds the permitted use, you will need to obtain permission directly from the copyright holder. To view a copy of this licence, visit http://creativecommons.org/licenses/by$\mathrm{nc} / 4.0 /$.

\section{REFERENCES}

1. Farag SS, Archer KJ, Mrozek K, et al. Pretreatment cytogenetics add to other prognostic factors predicting complete remission and long-term outcome in patients 60 years of age or older with acute myeloid leukemia: results from cancer and leukemia group B 8461. Cancer and Leukemia Group B 8461. Blood. 2006;108:63-73.

2. Fröhling S, Schlenk RF, Kayser S, et al. Cytogenetics and age are the major determinants of outcome in intensively treated acute myeloid leukemia patients older than 60 years: results from the AMLSG trial AML HD98-B. Blood. 2006;108:3280-8.

3. Balducci L. Aging, frailty, and chemotherapy. Cancer Control. 2007;14:7-12.

4. Klepin HD, Balducci L. Acute myelogenous leukemia in older adults. Oncologist. 2009;14:222-32. 
5. Godwin JE, Smith SE. Acute myeloid leukemia in the older patient. Crit Rev Oncol Hematol. 2003;48: S17-26.

6. Grimwade D, Walker $H$, Harrison $G$, et al. The predictive value of hierarchical cytogenetic classification in older adults with acute myeloid leukemia (AML): analysis of 1065 patients entered into the United Kingdom Medical Research Council AML11 trial. Blood. 2001;98:1312-20.

7. Leith CP, Kopecky KJ, Godwin J, et al. Acute myeloid leukemia in the elderly: assessment of multidrug resistance (MDR1) and cytogenetics distinguishes biologic subgroups with remarkably distinct responses to standard chemotherapy. A Southwest Oncology Group study. Blood. 1997;89:3323-9.

8. Löwenberg B, Ossenkoppele GJ, van Putten W, et al. High-dose daunorubicin in older patients with acute myeloid leukemia. N Engl J Med. 2009;361: 1235-48.

9. Ossenkoppele GJ, Graveland WJ, Sonneveld P, et al. The value of fludarabine in addition to ARA-C and G-CSF in the treatment of patients with high-risk myelodysplastic syndromes and AML in elderly patients. Blood. 2004;103:2908-13.

10. Willemze R, Suciu S, Meloni G, et al. High-dose cytarabine in induction treatment improves the outcome of adult patients younger than age 46 years with acute myeloid leukemia: results of the EORTC-GIMEMA AML-12 trial. J Clin Oncol. 2014;32:219-28.

11. Morra E, Barosi G, Bosi A, et al. Clinical management of primary non-acute promyelocytic leukemia acute myeloid leukemia: practice Guidelines by the Italian Society of Hematology, the Italian Society of Experimental Hematology, and the Italian Group for Bone Marrow Transplantation. Haematologica. 2009;94:102-12.

12. O'Donnell MR, Tallman MS, Abboud CN, et al. Acute myeloid leukemia, version 2.2013. J Natl Compr Canc Netw. 2013;11:1047-55.

13. Olivieri A, Capelli D, Troiani E, et al. A new intensive induction schedule, including high-dose idarubicin, high-dose aracytin and amifostine, in older AML patients: feasibility and long-term results in 42 patients. Exp Hematol. 2007;35:1074-82.

14. Mandelli F, Vignetti M, Suciu S, et al. Daunorubicin versus mitoxantrone versus idarubicin as induction and consolidation chemotherapy for adults with acute myeloid leukemia: the EORTC and GIMEMA Groups Study AML-10. J Clin Oncol. 2009;27: 5397-403.
15. Pautas C, Merabet F, Thomas X, et al. Randomized study of intensified anthracycline doses for induction and recombinant interleukin-2 for maintenance in patients with acute myeloid leukemia age 50 to 70 years: results of the ALFA-9801 study. J Clin Oncol. 2010;28:808-14.

16. Etienne A, Esterni B, Charbonnier A, et al. Comorbidity is an independent predictor of complete remission in elderly patients receiving induction chemotherapy for acute myeloid leukemia. Cancer. 2007;109:1376-83.

17. Repetto L, Fratino L, Audisio RA, et al. Comprehensive geriatric assessment adds information to Eastern Cooperative Oncology Group performance status in elderly cancer patients: an Italian Group for Geriatric Oncology study. J Clin Oncol. 2002;20: 494-502.

18. Flood $\mathrm{KL}$, Carrll $\mathrm{MB}$, Le $\mathrm{CV}$, et al. Geriatric sindrome in elderly patients admitted to an oncologyacute care for elders unit. J Clin Oncol. 2006;24: 2298-303.

19. Balducci L, Extermann M. Management of cancer in the older person: a practical approach. Oncologist. 2000;5:224-37.

20. Olivieri A, Gini G, Bocci C, et al. Tailored therapy in an unselected population of 91 elderly patients with DLBCL prospectively evaluated using a simplified CGA. Oncologist. 2012;17:663-72.

21. Slovak ML, Kopecky KJ, Wolman SR, et al. Cytogenetic correlation with disease status and treatment outcome in advanced stage leukemia post bone marrow transplantation: a Southwest Oncology Group study (SWOG-8612). Leuk Res. 1995;19: 381-8.

22. Capelli D, Chiarucci M, Poloni A, et al. Mobilization-driven postconsolidation therapy in elderly patients with acute myeloid leukemia: feasibility and efficacy of autologous stem cell transplantation versus low-dose gemtuzumab ozogamicin. Biol Blood Marrow Transplant. 2014;20:1399-406.

23. Cheson BD, Cassileth PA, Head DR, et al. Report of the National Cancer Institute-sponsored workshop on definitions of diagnosis and response in acute myeloid leukemia. J Clin Oncol. 1990;8:813-9.

24. Simon R. Optimal two-stage designs for phase II clinical trials. Control Clin Trials. 1989;10:1-10.

25. Amadori S, Suciu S, Jehn U, et al. Use of glycosylated recombinant human G-CSF (lenograstim) during and/or after induction chemotherapy in patients 61 years of age and older with acute myeloid leukemia: final results of AML-13, a randomized phase-3 study. Blood. 2005;106:27-34. 
26. Rowe JM, Kim HT, Cassileth PA, et al. Adult patients with acute myeloid leukemia who achieve complete remission after 1 or 2 cycles of induction have a similar prognosis: a report on 1980 patients registered to 6 studies conducted by the Eastern Cooperative Oncology Group. Cancer. 2010;116: 5012-21.

27. Appelbaum FR, Kopecky KJ. Long-term survival after chemotherapy for acute myeloid leukemia: the experience of the Southwest Oncology Group. Cancer. 1997;80:2199-204.

28. Malfuson JV, Etienne A, Turlure P, et al. Risk factors and decision criteria for intensive chemotherapy in older patients with acute myeloid leukemia. Haematologica. 2008;93:1806-13.

29. Sorror ML, Sandmaier BM, Storer BE, et al. Comorbidity and disease status based risk stratification of outcomes among patients with acute myeloid leukemia or myelodysplasia receiving allogeneic hematopoietic cell transplantation. J Clin Oncol. 2007;25:4246-54.

30. Kaplan EL, Meier P. Nonparametric estimation from incomplete observations. J Am Stat Assoc. 1958;53: 457-65.

31. Wedding U, Röhrig B, Klippstein A, et al. Impairment in functional status and survival in patients with acute myeloid leukaemia. J Cancer Res Clin Oncol. 2006;132:665-71.

32. Weiss MA, Aliff TB, Tallman MS, et al. A single, high dose of idarubicin combined with cytarabine as induction therapy for adult patients with recurrent or refractory acute lymphoblastic leukemia. Cancer. 2002;95:581-7.

33. Poloni A, Capelli D, Trappolini S, et al. Low-dose gemtuzumab-ozogamicin as post-consolidation therapy in elderly patients with acute myeloid leukaemia: a pilot study. Br J Haematol. 2010;150: 119-21.

34. Capelli D, Santini G, De Souza C, et al. Amifostine can reduce mucosal damage after high-dose melphalan conditioning for peripheral blood progenitor cell autotransplant: a retrospective study. Br J Haematol. 2000;110:300-7.

35. Olivieri A, Capelli D, Montanari M, et al. Very low toxicity and good quality of life in 48 elderly patients autotransplanted for hematological malignancies: a single center experience. Bone Marrow Transplant. 2001;27:1189-95.

36. Döhner H, Estey EH, Amadori S, European LeukemiaNet, et al. Diagnosis and management of acute myeloid leukemia in adults: recommendations from an international expert panel, on behalf of the European LeukemiaNet. Blood. 2010;115:453-74.

37. Lowenthal RM, Bradstock KF, Matthews JP, et al. A phase I/II study of intensive dose escalation of cytarabine in combination with idarubicin and etoposide in induction and consolidation treatment of adult acute myeloid leukemia. Australian Leukemia Study Group. Leuk Lymphoma. 1999;34: 501-10.

38. Bradstock KF, Matthews JP, Lowenthal RM, et al. A randomized trial of high-versus conventional-dose cytarabine in consolidation chemotherapy for adult de novo acute myeloid leukemia in first remission after induction therapy containing high-dose cytarabine. Blood. 2005;105:481-8.

39. Appelbaum FR, Gundacker H, Head DR, et al. Age and acute myeloid leukemia. Blood. 2006;107: 3481-5.

40. Castaigne S, Pautas C, Terré C, et al. Effect of gemtuzumab ozogamicin on survival of adult patients with de-novo acute myeloid leukaemia (ALFA0701): a randomised, open-label, phase 3 study. Lancet. 2012;379:1508-16.

41. Arellano M, Winton E, Pan L, et al. High-dose cytarabine induction is well tolerated and active in patients with de novo acute myeloid leukemia older than 60 years. Cancer. 2012;118:428-33.

42. Clavio M, Vignolo L, Albarello A, et al. Adding lowdose gemtuzumab ozogamicin to fludarabine, Ara$\mathrm{C}$ and idarubicin (MY-FLAI) may improve diseasefree and overall survival in elderly patients with non-M3 acute myeloid leukaemia: results of a prospective, pilot, multi-centre trial and comparison with a historical cohort of patients. Br J Haematol. 2007;138:186-95.

43. Candoni A, Martinelli G, Toffoletti E, et al. Gemtuzumab-ozogamicin in combination with fludarabine, cytarabine, idarubicin (FLAI-GO) as induction therapy in CD33-positive AML patients younger than 65 years. Leuk Res. 2008;32:1800-8.

44. Ferrara F, D'Arco AM, De Simone M, et al. Fludarabine and cytarabine as continuous sequential infusion for elderly patients with acute myeloid leukemia. Haematologica. 2005;90:776-84.

45. Kantarjian H, Ravandi F, O'Brien S, et al. Intensive chemotherapy does not benefit most older patients (age 70 years or older) with acute myeloid leukemia. Blood. 2010;116:4422-9.

46. Burnett AK, Russell NH, Hunter AE, et al. Clofarabine doubles the response rate in older patients with acute myeloid leukemia but does not improve survival. Blood. 2013;122:1384-94. 
47. Faderl S, Wetzler M, Rizzieri D, et al. Clofarabine plus cytarabine compared with cytarabine alone in older patients with relapsed or refractory acute myelogenous leukemia: results from the CLASSIC I Trial. J Clin Oncol. 2012;30:2492-9.

48. Dombret H, Seymour JF, Butrym A, et al. International phase 3 study of azacitidine vs conventional care regimens in older patients with newly diagnosed AML with $>30 \%$ blasts. Blood. 2015;126: 291-9.

49. Kantarjian HM, Thomas XG, Dmoszynska A, et al, Arthur C. Multicenter, randomized, open-label, phase III trial of decitabine versus patient choice, with physician advice, of either supportive care or low-dose cytarabine for the treatment of older patients with newly diagnosed acute myeloid leukemia. J Clin Oncol. 2012;30:2670-7.

50. Thomas X, Suciu S, Rio B, et al. Autologous stem cell transplantation after complete remission and first consolidation in acute myeloid leukemia patients aged 61-70 years: results of the prospective EORTC-GIMEMA AML-13 study. Haematologica. 2007;92:389-96.

51. Oriol A, Ribera JM, Esteve J, et al. Feasibility and results of autologous stem cell transplantation in de novo acute myeloid leukemia in patients over 60 years old. Results of the CETLAM AML-99 protocol. Haematologica. 2004;89:791-800.
52. Scholl S, Theuer C, Scheble V, et al. Clinical impact of nucleophosmin mutations and Flt3 internal tandem duplications in patients older than $60 \mathrm{yr}$ with acute myeloid leukaemia. Eur J Haematol. 2008;80:208-15.

53. Röllig C, Bornhäuser $\mathrm{M}$, Thiede $\mathrm{C}$, et al. Long-term prognosis of acute myeloid leukemia according to the new genetic risk classification of the European LeukemiaNet recommendations: evaluation of the proposed reporting system. Blood. 2010;115: 453-74.

54. Sorror M, Giralt S, Sandmaier B, et al. Hematopoietic cell transplantation-specific comorbidity index as an outcome predictor for patients with acute myeloid leukemia in first remission: combined FHCRC and MDACC experiences. Blood. 2007;110: 4606-13.

55. Löwenberg B, Beck J, Graux C, et al. Gemtuzumab ozogamicin as postremission treatment in AML at 60 years of age or more: results of a multicenter phase 3 study. Blood. 2010;115:2586-91.

56. Itzykson R, Gardin C, Pautas C, et al. Impact of post-remission therapy in patients aged $65-70$ years with de novo acute myeloid leukemia: a comparison of two concomitant randomized ALFA trials with overlapping age inclusion criteria. Haematologica. 2011;96:837-44. 